\title{
Autoassociative Fuzzy Implicative Memory on the Platform of Fuzzy Preorder
}

\author{
Irina Perfilieva ${ }^{1}$ Marek Vajgl $^{1}$ \\ ${ }^{1}$ IRAFM, University of Ostrava, 30. dubna 22, Ostrava, Czech Republic
}

\begin{abstract}
We propose a new theoretical justification of implicative fuzzy associative memory. It is based on the notion of a fuzzy preorder operator. Moreover, we give a necessary and sufficient condition on input data that guarantees insensitivity to a certain type of noise. The latter is precisely characterized. Various illustrative examples are made.
\end{abstract}

Keywords: fuzzy preorder, upper set, fuzzy associative memory, image processing, noise

\section{Introduction}

Associative memory is a model based on the biological phenomena with the aim to storage the input data and retrieve it even in the case of damaged, incomplete or noisy defects. Implicative fuzzy associative memory (IFAM) is a particular case of a fuzzy associative memory that is based on the implicative form of synaptic weights, see e.g. [1, 3, 12].

Associative memory mechanism has been proposed in the papers of Taylor and Steinbuch on matrix associative memories $[13,11]$. The first attempt to construct a fuzzy associative memory (FAM) has been made by Kosko - [4]. This approach presented FAM as a single-layer feedforward neural net containing nonlinear matrix-vector product. This approach was later extended with the purpose to increase a storage capacity (e.g. [2]). Significant progress was achieved by the introduction of the so called learning implication rules $[1,3]$, that afterwards lead to implicative fuzzy associative memory (IFAM) with implicative fuzzy learning. In [12], theoretical backgrounds of IFAM were discussed and examples that demonstrate the ability of IFAM to work with incomplete and noisy information were given.

In our contribution, we give another theoretical justification of IFAM that uses the notion and properties of a fuzzy preorder relation. This enables us to discover conditions on input data that guarantee that IFAM works properly. Moreover, we found a necessary and sufficient condition on input data that ensures insensitivity to a certain type of noise.

\section{Preliminaries}

\subsection{Implicative fuzzy associative memory}

In this section, we remind backgrounds of the theory of fuzzy associative memories and their implicative forms as they were presented in [12]. Generally speaking, to design a fuzzy associative memory means to find a fuzzy relation that connects a given finite set of inputs with a given finite set of outputs. The term associative reflects the ability of capturing an association between inputs and outputs.

We assume that fuzzy associative memory is a one level neural network endowed with Pedrycz's neurons with thresholds, whose input-output relationship can be characterized by

$$
\mathbf{y}_{i}=\bigvee_{j=1}^{n}\left(w_{i j} \mathrm{t} \mathbf{x}_{j}\right) \vee \theta_{i}, i=1, \ldots, m,
$$

where all elements are from $[0,1], t$ is a $t$-norm, $W=$ $\left(w_{i j}\right)$ is a connecting fuzzy relation or (in terms of neural networks) is a $m \times n$ synaptic weight matrix, $\theta$ is a threshold (bias), $m$ represents the length of data and $n$ represents the number of constituent input patterns.

Implicative fuzzy associative memory [12] has the connecting fuzzy relation $W$ in the form that uses the adjoint to $t$ implication $\rightarrow$ :

$$
w_{i, j}=\bigwedge_{k=1}^{p}\left(\mathbf{x}_{j}^{k} \rightarrow \mathbf{y}_{i}^{k}\right),
$$

where $\left(\mathbf{x}^{k}, \mathbf{y}^{k}\right), k=1, \ldots, p$, are couples of inputoutput patterns.

The IFAM, considered in this article, is based on the Łukasiewicz t-norm $a$ t $b=\max (0, a+b-1)$, and the adjoint implication $a \rightarrow b=\min (1,1-a+$ b).

In autoassociative fuzzy implicative memory (AFIM) the input-output patterns are the same objects (images).

Let $\left\{\mathbf{x}^{1}, \ldots, \mathbf{x}^{p}\right\}$ be a database of image patterns, each is represented by a $n$-dimensional vector of pixels. The weight matrix $W$ of AFIM is defined in (2), and the bias vector is calculated as follows:

$$
\theta_{i}=\bigwedge_{k=1}^{p} \mathbf{x}_{i}^{k}
$$

Then AFIM returns output image $\mathbf{y}$ for the input given by $\mathbf{x}$ in accordance with (1). 
One of the main benefits of AFIM is its error correction ability. In [12], it has been claimed that an eroded noise can be removed, where an eroded version of $\mathbf{x}^{k}$ is any image $\tilde{\mathbf{x}}^{k}$ that fulfills $\tilde{\mathbf{x}}^{k} \leq \mathbf{x}^{k}$. Our critical remark to this claim is that the zero eroded version of any input pattern cannot be successfully reconstructed, if a retrieval procedure is based on (1). Therefore, a careful study of the retrieval mechanism is required.

In the proposed contribution, we analyze the AFIM retrieval mechanism with respect to two abilities: (a) to return the output that is identical to a certain input from a database; (b) to remove a noise. We find a necessary and sufficient condition on input patterns and characterize a noise that can be successfully removed by the retrieval procedure.

\subsection{Algebraic background}

Let $\mathcal{L}=\langle L, \vee, \wedge, *, \rightarrow, 0,1\rangle$ be a fixed, complete, integral, residuated, commutative l-monoid (a complete residuated lattice). Let $X$ be a non-empty set, $L^{X}$ a class of fuzzy sets on $X$ and $L^{X \times X}$ a class of fuzzy relations on $X$. Fuzzy sets and fuzzy relations are identified with their membership functions, i.e. elements from $L^{X}$ and $L^{X \times X}$, respectively. A fuzzy set $A$ is normal if there exists $x_{A} \in X$ such that $A\left(x_{A}\right)=1$. The (ordinary) set $\operatorname{Core}(A)=\{x \in X \mid A(x)=1\}$ is the core of the normal fuzzy set $A$. Fuzzy sets $A \in L^{X}$ and $B \in L^{X}$ are equal $(A=B)$, if for all $x \in X, A(x)=B(x)$. A fuzzy set $A \in L^{X}$ is less than or equal to a fuzzy set $B \in L^{X}(A \leq B)$, if for all $x \in X, A(x) \leq B(x)$.

The lattice operations $\vee$ and $\wedge$ induce the union and intersection of fuzzy sets, respectively. The binary operation $*$ of $\mathcal{L}$ is used below for set-relation composition of the type sup-*, which is usually denoted by $\circ$.

$$
(A \circ R)(y)=\bigvee_{x \in X}(A(x) * R(x, y)) .
$$

The o composition was introduced by L. Zadeh [15] in the form $\max -\min$.

\section{Fuzzy Preorders and Their Eigen Sets}

We will first recall basic facts about fuzzy preorder relations as they were presented in [5]. Then we will characterize eigen sets of fuzzy preorder relations and how they can be reconstructed.

\subsection{Fuzzy preorders and their upper and lower sets}

The text in this subsection is an adapted version of [6].

A binary fuzzy relation on $X$ is a $*$-fuzzy preorder of $X$ if it is reflexive and $*$-transitive. The fuzzy preorder $Q^{*} \in L^{X \times X}$, where

$$
Q^{*}(x, y)=\bigwedge_{i \in I}\left(A_{i}(x) \rightarrow A_{i}(y)\right),
$$

is generated by an arbitrary family of fuzzy sets $\left(A_{i}\right)_{i \in I}$ of $X$.

If $Q$ is a fuzzy preorder on $X$, then the fuzzy set $A \in L^{X}$ such that

$$
\begin{array}{r}
A(x) * Q(x, y) \leq A(y) \quad(A(y) * Q(x, y) \leq A(x)), \\
x, y \in X,
\end{array}
$$

is called an upper set (a lower set) of $Q$ (see [5]). Denote $Q^{t}(x)=Q(t, x)\left(Q_{t}(x)=Q(x, t)\right), x \in X$, and see that $Q^{t}\left(Q_{t}\right)$ is an upper set (lower set) of $Q$. The fuzzy set $Q^{t}\left(Q_{t}\right)$ is called a principal upper set (lower set).

If $Q$ is a fuzzy preorder on $X$, then $Q^{o p} \in L^{X \times X}$ such that $Q^{o p}(x, y)=Q(y, x)$ is a fuzzy preorder on $X$ as well. It follows that an upper set of $Q$ is a lower set of $Q^{o p}$ and vice versa. For this reason, our results will be formulated for upper sets of respective fuzzy preorders.

The necessary and sufficient condition that a family of fuzzy sets of $X$ constitutes a family of upper sets of some fuzzy preorder on $X$ has been proven in [5]. In Theorem 1 [6], given below, we characterize principal upper sets of a fuzzy preorder on $X$. Let us remark that assumptions of Theorem 1 are different from those in [5].

\section{Theorem 1}

Let $I$ be an index set, $\left(A_{i}\right)_{i \in I} \subseteq L^{X}$ a family of normal fuzzy sets of $X$ and $\left(x_{i}\right)_{i \in I} \subseteq X$ a family of pairwise different core elements such that for all $i \in I, A_{i}\left(x_{i}\right)=1$. Then the following statements are equivalent:

(i) There exists a fuzzy preorder $Q$ on $X$ such that for all $i \in I, x \in X, A_{i}(x)=Q\left(x_{i}, x\right)\left(A_{i}\right.$ is a principal upper set of $Q)$.

(ii) For all $i \in I, x \in X, A_{i}(x)=Q^{*}\left(x_{i}, x\right)\left(A_{i}\right.$ is a principal upper set of $\left.Q^{*}\right)$ where $Q^{*}$ is given by (3).

(iii) For all $i, j \in I$,

$$
A_{i}\left(x_{j}\right) \leq \bigwedge_{x \in X}\left(A_{j}(x) \rightarrow A_{i}(x)\right) .
$$

\section{Corollary 1}

Let $\left(A_{i}\right)_{i \in I} \subseteq L^{X}$ be a family of normal fuzzy sets of $X$ and $\left(x_{i}\right)_{i \in I} \subseteq X$ a family of pairwise different core elements such that for all $i, j \in I$, (4) holds true. Then $Q^{*}$ is the coarsest fuzzy preorder on $X$ such that every fuzzy set $A_{i}, i \in I$, is a principal upper set of $Q^{*}$.

\section{Remark 1}

On the basis of Theorem 1 and its Corollary 1, we conclude that a family of normal fuzzy sets $\left(A_{i}\right)_{i \in I} \subseteq L^{X}$ with pairwise different core elements $\left(x_{i}\right)_{i \in I} \subseteq X$, such that (4) is fulfilled, generates the coarsest fuzzy preorder $Q^{*}$ on $X$ such that every family element $A_{i}$ is a principal upper set of $Q^{*}$ that corresponds to its core element $x_{i}$. 


\section{Remark 2}

The fuzzy preoreder $Q^{*}$ (3) is often called the Valverde order on $X$ determined by a family of fuzzy sets $\left(A_{i}\right)_{i \in I}$ of $X$ (see [14] for details).

\subsection{Eigen sets of fuzzy preorders}

In this subsection, we show that if the assumptions of Theorem 1 are fulfilled, and if fuzzy preorder $Q^{*}$ on $X$ is generated by normal fuzzy sets $\left(A_{i}\right)_{i \in I} \subseteq$ $L^{X}$ with pairwise different core elements $\left(x_{i}\right)_{i \in I} \subseteq$ $X$ (in the sense of (3)), then these fuzzy sets are so called eigen sets (see [10] for background), i.e. they fulfill

$$
A_{i} \circ Q^{*}=A_{i}, i \in I .
$$

\section{Proposition 1}

Let family $\left(A_{i}\right)_{i \in I} \subseteq L^{X}, i \in I$, of normal fuzzy sets of $X$ with pairwise different core elements $\left(x_{i}\right)_{i \in I} \subseteq$ $X$ fulfill (4) and generate fuzzy preorder $Q^{*}$ in the sense of (3). Then every $A_{i}, i \in I$, is an eigen set of $Q^{*}$.

PROOF: Let us choose and fix $A_{i}, i \in I$. By Theorem $1, A_{i}(x)=Q^{*}\left(x_{i}, x\right)$. Then

$$
\begin{aligned}
& A_{i}(x) \circ Q^{*}(x, y)=\bigvee_{x \in X}\left(Q^{*}\left(x_{i}, x\right) * Q^{*}(x, y)\right) \leq \\
& Q^{*}\left(x_{i}, y\right)=A_{i}(y)
\end{aligned}
$$

On the other hand,

$$
\begin{aligned}
& A_{i}(x) \circ Q^{*}(x, y)=\bigvee_{x \in X}\left(Q^{*}\left(x_{i}, x\right) * Q^{*}(x, y)\right) \geq \\
& Q^{*}\left(x_{i}, y\right) * Q^{*}(y, y)=Q^{*}\left(x_{i}, y\right)=A_{i}(y)
\end{aligned}
$$

\section{Corollary 2}

Let the assumptions of Propositions 1 be fulfilled and fuzzy set $\bar{A}_{i} \in L^{X}, i \in I$, be a "skeleton" of $A_{i}$, (we will call it $\{0,1\}$-reduction) where

$$
\bar{A}_{i}(x)= \begin{cases}1, & \text { if } x \in \operatorname{Core}\left(A_{i}\right) \\ 0, & \text { otherwise }\end{cases}
$$

Then $A_{i}$ can be reconstructed from $\bar{A}_{i}$, i.e.

$$
\bar{A}_{i} \circ Q^{*}=A_{i}
$$

PROOF: By Proposition 1, and because $\bar{A}_{i} \leq A_{i}$, we have $\bar{A}_{i} \circ Q^{*} \leq A_{i} \circ Q^{*}=A_{i}$. On the other hand,

$$
\begin{array}{r}
\left(\bar{A}_{i} \circ Q^{*}\right)(y)=\bigvee_{x \in X}\left(\bar{A}_{i}(x) * Q^{*}(x, y)\right) \geq Q^{*}\left(x_{i}, y\right)= \\
A_{i}(y) .
\end{array}
$$

\section{Corollary 3}

Let the assumptions of Propositions 1 be fulfilled and fuzzy set $\widetilde{A}_{i} \in L^{X}$ be "in between" $\bar{A}_{i}$ and $A_{i}$, i.e.

$$
\bar{A}_{i} \leq \widetilde{A}_{i} \leq A_{i},
$$

where $i \in I$. Then $A_{i}$ can be reconstructed from $\widetilde{A}_{i}$, i.e.

$$
\widetilde{A}_{i} \circ Q^{*}=A_{i}
$$

PROOF: The proof follows from the following chain of inequalities:

$$
A_{i}=A_{i} \circ Q^{*} \geq \widetilde{A}_{i} \circ Q^{*} \geq \bar{A}_{i} \circ Q^{*}=A_{i} .
$$

The following proposition is important for the below considered applications. It shows that under the assumptions of Propositions 1 , every $A_{i}, i \in I$, is an eigen set of another fuzzy preorder $Q^{r}$, which is composed from all these constituent fuzzy sets. By saying "composed", we mean that opposite to $Q^{*}$, $Q^{r}$ does not require any computation.

\section{Proposition 2}

Let family $\left(A_{i}\right)_{i \in I} \subseteq L^{X}, i \in I$, of normal fuzzy sets of $X$ with pairwise different core elements $\left(x_{i}\right)_{i \in I} \subseteq$ $X$ fulfill (4). Then every $A_{i}, i \in I$, is an eigen set of the following fuzzy preorder

$$
Q^{r}(x, y)= \begin{cases}A_{i}(y), & \text { if } x=x_{i} \\ 1, & \text { if } x=y, \\ 0, & \text { otherwise }\end{cases}
$$

\section{Fuzzy Preorders and AFIM}

In this section, we will put a bridge between the theory, presented in Section 3, and the theory of $\mathrm{Au}-$ toassociative Fuzzy Implicative Memories (AFIM), presented in [12]. We will see that in the proposed below model of AFIM, a connecting fuzzy relation (synaptic weight matrix) is a fuzzy preorder relation.

In details, we choose $L=[0,1]$, Eukasiewicz algebra, and database $\left\{\mathbf{x}^{1}, \ldots, \mathbf{x}^{p}\right\}$ of fuzzy images, where every particular image is a mapping $\mathbf{x}^{k}: X \rightarrow[0,1], X=\{1, \ldots, n\}$, that corresponds to a normal fuzzy set. In accordance with (3), we construct the fuzzy preorder relation $Q^{*}$, such that

$$
Q^{*}(i, j)=\bigwedge_{k=1}^{p}\left(\mathbf{x}^{k}(i) \rightarrow \mathbf{x}^{k}(j)\right) .
$$

We remark that this is the reverse fuzzy preorder with respect to that given by (2). In the terminology of the theory of Autoassociative Memories, the results from Section 3 show that under condition (4),

- each constituent input image $\mathbf{x}^{k}, k=1, \ldots, p$, can be retrieved, if the synaptic weight matrix $W$ is equal to $Q^{*}$ and the computation of the output is based on the simpler version of (1), i.e.

$$
\mathbf{y}_{i}=\bigvee_{j=1}^{n}\left(\mathbf{x}_{j}^{k} \mathrm{t} w_{i j}\right), i=1, \ldots, m,
$$

which does not involve bias $\theta$;

- each constituent input image $\mathbf{x}^{k}, k=1, \ldots, p$, can be retrieved, if the synaptic weight matrix $W$ is equal to $Q^{r}$ (see (9)) with the subsequent computation of the output by (10); 
- each constituent input image $\mathbf{x}^{k}, k=1, \ldots, p$, can be fully reconstructed from its binary "skeleton" (see (6) in Corollary 2).

Let us remark that from the second result, listed above, it follows that there is no need in a computation of the synaptic weight matrix $W$, because it can be assembled from constituent input images in accordance with (9). This fact leads to a tremendous saving of computational complexity.

Moreover, from Corollary 3 we can deduce a complete characterization of a noise $N_{i}$ that can be "added to" (actually, subtracted from) a constituent input image $\mathrm{x}^{i}$ without any corruption of the output. In details,

$$
N(t)= \begin{cases}n(t), & \text { if } t \notin \text { Core }^{i} \\ 0, & \text { otherwise }\end{cases}
$$

where for $t \notin C$ Core $\mathbf{x}^{i}$, the value $n(t)$ fulfills the requirement $0 \leq n(t) \leq \mathbf{x}^{i}(t)$.

In the section below, we demonstrate how the presented above theory works in the case of benchmark input images. We will illustrate all the above listed results.

\section{Illustration}

The aim of this section is to give illustrations to the theoretical results of this paper. We use the gray scale with the range $[0,1]$, where 0 (1) represents the black (white) color. We chose two sets of databases, both were artificially created from available ones. The sets contain $2 \mathrm{D}$ images of $20 \times 20$ and $32 \times 32$ pixels, respectively, where all images are represented by vectors that are comprised by successive rows. Each image corresponds to a fuzzy set on $\{1, \ldots, 20\} \times\{1, \ldots, 20\}$ or $\{1, \ldots, 32\} \times\{1, \ldots, 32\}$ with values in $[0,1]$.

\subsection{Experiments with abstract images}

We have created three databases A, B, and C of $2 \mathrm{D}$ images of $20 \times 20$ pixels, where condition (4) is/is not fulfilled, details are below.

- Database A - contains three images (see figure 1) such that (4) is fulfilled.

- Database B - contains four images (see figure 2) such that (4) is not fulfilled with the nonseparability degree as follows:

$$
D_{B}=\bigwedge_{x \in X}\left(A_{j}(x) \rightarrow A_{i}(x)\right) \rightarrow A_{i}\left(x_{j}\right) .
$$

- Database C - contains eight images (see figure 3) such that (4) is not fulfilled, and the corresponding non-separability degree $D_{C}$ is less than $D_{B}$.

For each database of images, we computed corresponding fuzzy preorder $Q^{*}$ and its reduction $Q^{r}$.

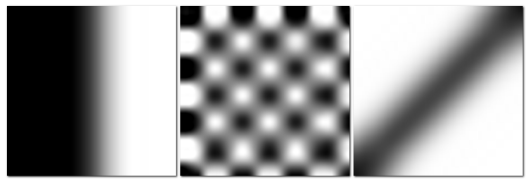

Figure 1: Database A contains three images such that (4) is fulfilled.

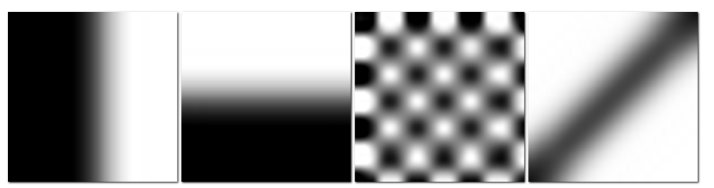

Figure 2: Database B contains four images such that (4) is not fulfilled with the degree $D_{B}$.

In Fig. 4, we demonstrate the influence of condition (4) on the quality of retrieval by the AFIM mechanism, where the computation of the output is based on (10) and the synaptic weight matrix $W$ is equal to $Q^{*}$ or $Q^{r}$. For this purpose we choose the third image from database $\mathrm{A}$ as an input (Fig. 4a) and retrieve it from each of three databases A, B and C. In Fig. 4c, we see that if the synaptic weight matrix $W$ is computed (comprised) from database A as fuzzy preorder $Q^{*}\left(Q^{r}\right)$, then the output is identical with the input. If $W$ is computed from database $\mathrm{C}$ as fuzzy preorder $Q^{*}$, then the output differs from the input. In Fig. 4c, we show the binary "skeleton" of the third image from database A. In (Fig. 4c), we verified that if $W$ is computed/comprised from database A as fuzzy preorder $Q^{*} / Q^{r}$, then both outputs of the "skeleton" input is identical with its "full" prototype. In Fig. 5, we demonstrate how eroded noise (11) can be removed by the AFIM retrieval. We added (Fig. 5a) $70 \%$ dense erosion to the third image from database $\mathrm{A}$ and process the obtained eroded image by the IFAM with the synaptic weight matrix $W$ that corresponds to fuzzy preorder $Q^{r}$, computed from database A. In (Fig. 5b), we show the. In the next experiment, we wanted to show the influence of the non-separability degree. For this purpose, we considered databases B and C and computed average RMSEs' of outputs that correspond to all possible inputs. Our computation

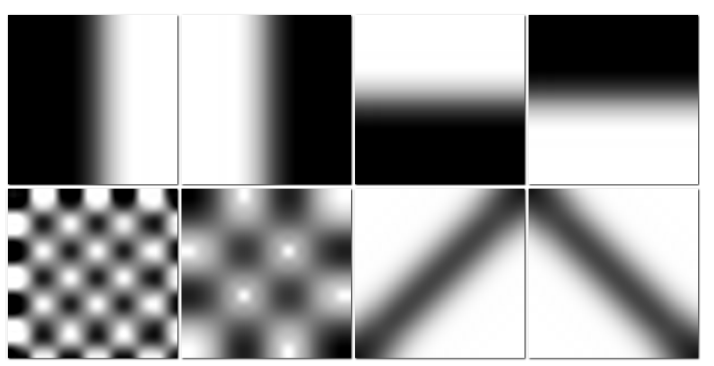

Figure 3: Database C contains eight images such that (4) is not fulfilled with the degree $D_{C}$ such that $D_{C} \leq D_{B}$. 


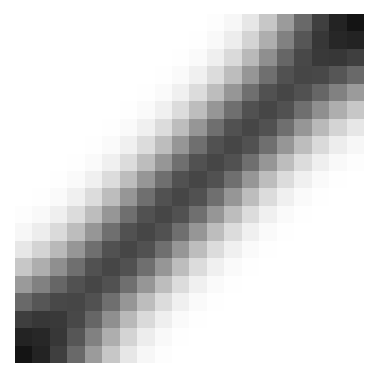

(a) Third image from (b) Binary "skeleton" of database A.

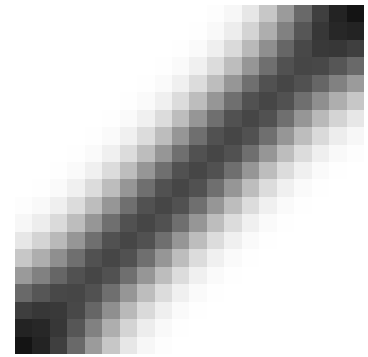
the third image from database A.

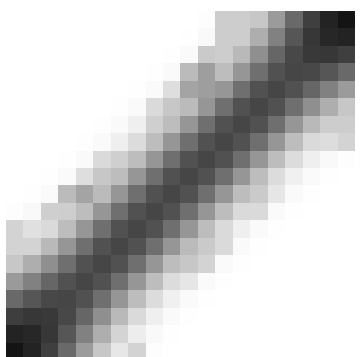

(c) Output image com- (d) Output image computed from database A - puted from database C identical with the input different from the input image. image.

Figure 4: Demonstration of the influence of condition (4) on the quality of retrieval by the AFIM mechanism. In database A, (4) is fulfilled, while in database C, (4) is not fulfilled.

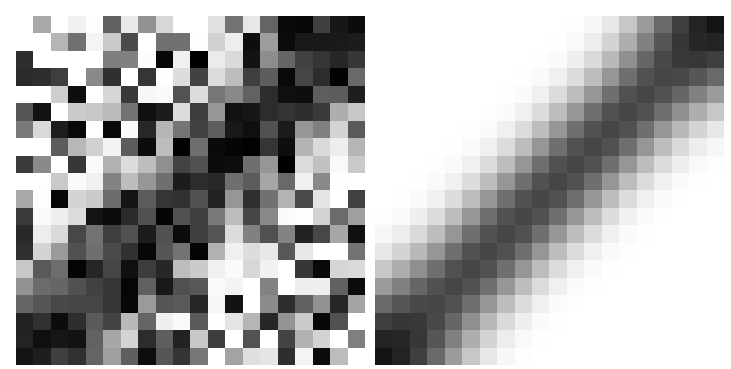

(a) Third image from (b) The output, retrieved database A with $70 \%$ of by IFAM with the synaperoded noise density. tic weight matrix $Q^{r}$.

Figure 5: Eroded noise has been removed by the AFIM retrieval.

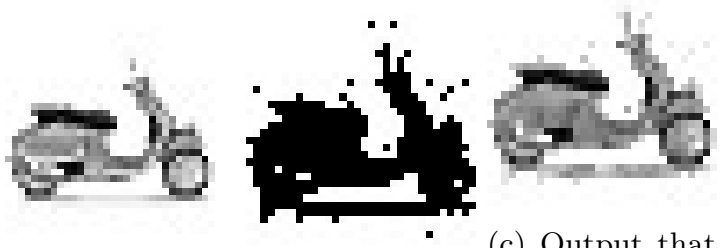

(a) Input image (b) Skeleton of corresponds "Moto" "Moto" the skeleton of "Moto"

Figure 6: Icon "Moto", its skeleton and output after processing by the proposed AFIM mechanism.

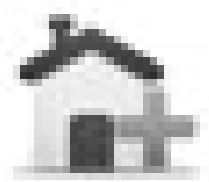

(a) Input image "House"
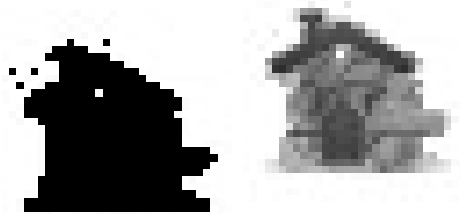

(c) Output that (b) Skeleton of corresponds to "House" the skeleton of "House"
Figure 7: Icon "House", its skeleton and output after processing by the proposed AFIM mechanism.

was based on (10) and the synaptic weight matrix $W$ was equal to $Q^{*}$. We obtained that the average RMSE for database B is equal to 0.000123, while the average RMSE for database $\mathrm{C}$ is equal to 0.001177 . This confirms our conclusion that the less is the non-separability degree, the worse is the retrieval quality measured by RMSE.

\subsection{Experiments with icon images}

In this section, we will be working with a database of 32 icons (size $32 \times 32$ ). We apply the proposed AFIM mechanism, where the computation of the output is based on (10) and the synaptic weight matrix $W$ is equal to $Q^{*}$. In order to discover, whether condition (4) is fulfilled, we choose some "skeleton" images that correspond to input ones and produce outputs in accordance with (10). In Fig. 6 and 7, we see that outputs of two "skeleton" images do not coincide with their "full" prototypes. Therefore in the chosen database, condition (4) is not fulfilled.

In order to increase the quality of retrieval by the same AFIM, we increase the core areas of input skeleton images. We will verify our conjecture that the larger is the core the better quality of retrieval can be obtained. For this purpose, we will introduce threshold $t \in[0,1]$ and create a set of skeleton images (for every $\mathbf{x}^{k}$ from the database) as follows:

$$
\mathbf{x}_{t}^{k}(u, v)= \begin{cases}1 \text { (white), } & \text { if } \mathbf{x}^{k}(u, v) \geq t \\ 0 \text { (black), } & \text { otherwise }\end{cases}
$$

In Fig. 8, we demonstrate how the quality of retrieval increases with the decrease of threshold $t$. 


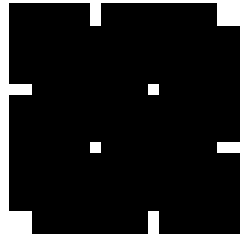

(a) Input, $t=1$

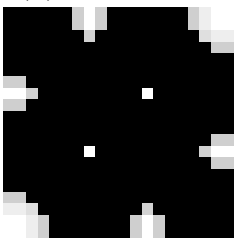

(d) Input, $t=0.8$

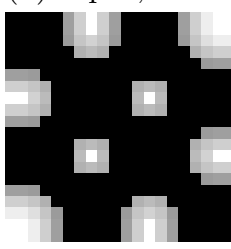

(g) Input, $\mathrm{t}=0.6$

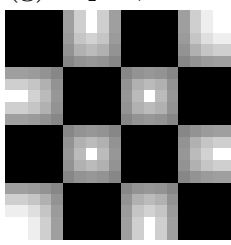

(j) Input, $\mathrm{t}=0.5$

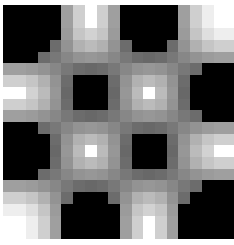

(k) Out, $\mathrm{t}=0.5$

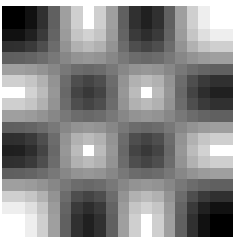

(m) Input, $\mathrm{t}=0.4$

(n) Out, $t=0.4$
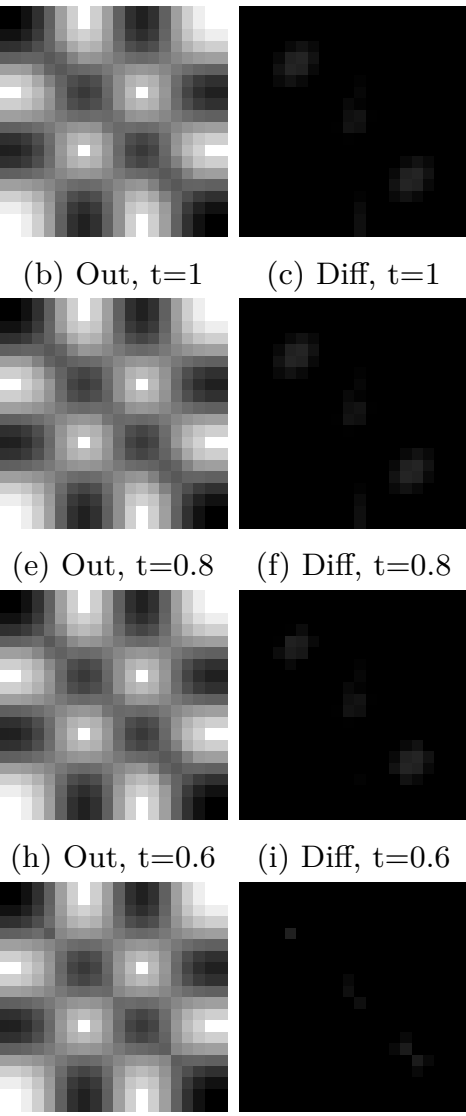

(c) Diff, $t=1$

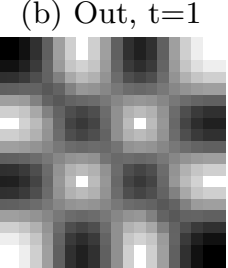

(e) Out, t $=0.8$
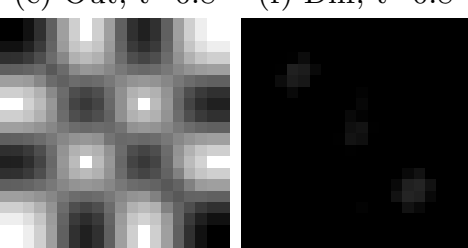

(i) Diff, $\mathrm{t}=0.6$

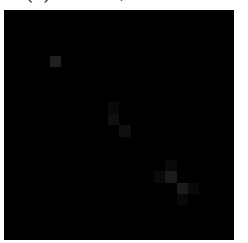

(1) Diff, $t=0.5$

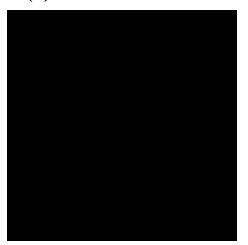

(o) Diff, $t=0.4$

Figure 8: Demonstration of impact of the core area on the quality of retrieval. Each input corresponds to a certain value of $t$, "Out" corresponds to the output image, "Diff" corresponds to the difference between the output and the original "full" image.

\section{Conclusion}

A new theoretical justification of implicative fuzzy associative memory has been proposed. We showed that the proposed mechanism processes every database image in such a way that it is successfully retrieved,

- if images in the database are well separated, the synaptic weight matrix $W$ corresponds to a certain fuzzy preorder relation and the computation of the output is based on a composition with $W$, which does not involve bias $\theta$;

- if different to the above conditions, the synaptic weight matrix $W$ corresponds to a certain reduction of the fuzzy preorder relation.
Moreover, we discovered a necessary and sufficient condition on database images that guarantees insensitivity to a certain type of noise. The latter is precisely characterized.

\section{References}

[1] Q. Cheng, Z.-T. Fan, "The stability problem for fuzzy bidirectional associative memories," Fuzzy Sets Syst., vol. 132 (2002), no. 1, pp. 83-90.

[2] F.-L. Chung, T. Lee, "On fuzzy associative memory with multiple-rule storage capacity,", IEEE Trans. Fuzzy Syst., vol. 4 (1996), no. 4, pp. 375384.

[3] F. Junbo, J. Fan, S. Yan, "A learning rule for fuzzy associative memories," Proc. IEEE Int. Conf. Neural Networks, IEEE World Congr. Computational Intelligence, vol. 7 (1994), pp. 4273-4277.

[4] B. Kosko, "Neural Networks and Fuzzy Systems: A Dynamical Systems Approach to Machine Intelligence," Englewood Cliffs, NJ: Prentice-Hall (1992).

[5] H. Lai, D. Zhang, "Fuzzy preorder and fuzzy topology," Fuzzy Sets and Syst., vol. 157 (2006), pp. $1865-1885$.

[6] I. Perfilieva, "Finitary solvability conditions for systems of fuzzy relation equations," Information Sciences 234 (2013), pp. 29-43.

[7] I. Perfilieva, "Fuzzy relation equations in semilinear spaces," Proc. Conf. IPMU'2010, Dortmund, Germany, pp. 545-552.

[8] I. Perfilieva, "Systems of fuzzy relation equations in a space with fuzzy preorder," Proc. of IFSAEUSFLAT 2009 Joint Conf., Lisbon, pp. 16011605.

[9] E. H. Ruspini, "A new approach to clustering," Information and Control 15 (1969), pp. 22-32.

[10] E. Sanchez, "Resolution of Eigen fuzzy sets equations", Fuzzy Sets and Syst., vol. 1 (1978), no. 1, pp. 69-74.

[11] K. Steinbuch, "Die lernmatrix," Kybernetik, vol. 1 (1961), pp. 36-45.

[12] P. Sussner, M.-E. Valle, "Implicative Fuzzy Associative Memories," IEEE Trans. Fuzzy Syst., vol. 14 (2006), pp. 793-807.

[13] W. Taylor, "Eletrical simulation on some nervous system functional activities,", Inf. Theory, vol. 15 , no. 1, pp. 166-177, Jan. 2004

[14] L. Valverde "On the structure of Findistinguishability operators", Fuzzy Sets and Syst., vol. 17, no. 3, pp. 313-328, Dec. 1985.

[15] L. A. Zadeh, The concept of a linguistic variable and its application to approximate reasoning I, II, III, Information Sciences 8-9 (1975), pp. 199-257, pp. 301-357, pp. 43-80. 